\title{
Altered Gene Expression Profiles in the Lungs of Streptozotocin-induced Diabetic Mice
}

\author{
Jung-Hyun Kim", Roya Rasaei ${ }^{1 \#}$, Sujin Park', Ji-Young Kim', Sunghun Na², \\ and ${ }^{\dagger}$ Seok-Ho Hong' \\ 'Dept. of Internal Medicine, School of Medicine, Kangwon National University, Chuncheon 24341, Korea \\ ${ }^{2}$ Dept. of Obstetrics and Gynecology, School of Medicine, Kangwon National University, Chuncheon 24341, Korea
}

\section{Abstract}

Diabetes mellitus is a common heterogeneous metabolic disorder, characterized by deposition of extracellular matrix, oxidative stress, and vascular dysfunction, thereby leading to gradual loss of function in multiple organs. However, little attention has been paid to gene expression changes in the lung under hyperglycemic conditions. In this study, we found that diabetes inuced histological changes in the lung of streptozotocin-induced diabetic mice. Global gene expression profiling revealed a set of genes that are up- and down-regulated in the lung of diabetic mice. Among these, expression of Amigo2, Adrb2, and Zbtb16 were confirmed at the transcript level to correlate significantly with hyperglycemia in the lung. We further evaluated the effect of human umbilical cord-derived perivascular stem cells (PVCs) on these gene expression in the lung of diabetic mice. Our results show that administration of PVC-conditioned medium significantly suppressed Amig2, Adrb2, and Zbtb16 upregulation in these mice, suggesting that these genes may be useful indicators of lung injury during hyperglycemia. Furthermore, PVCs offer a promising alternative cell therapy for treating diabetic complications via regulation of gene expression.

Keywords: Diabetes, Perivascular cells, Lung, Gene expression profile

\section{INTRODUCTION}

A chronic hyperglycemic condition has widespread adverse effects on various tissues, including the heart, kidney, retina, muscle, liver, and vasculature (Rask-Madsen et al., 2013; Bissel, 2015). Emerging evidence indicates that the functions and structure of the lung are also affected by diabetes mellitus (DM). Decline in total lung capacity, forced vital capacity, and diffusion capacity has been reported in patients with DM and may contribute to increased risk for pulmonary diseases (Ehrlich et al., 2010; Pitocco et al., 2012; Kolahian et al., 2019). Recent studies of DM patients and diabetic animal models demonstrated that DM induces fibrotic changes that accompany an epithelial-to-mesenchymal transition in the lung, which is mediated through the activation of transforming growth factor (TGF)- $\beta$ signaling pathways (Talakatta et al., 2018). Gene expression profiling in the lung of diabetic rats revealed altered expression of specific sets of genes related to apoptosis, the stress response, and collagen (Lunteren et al., 2014). However, the molecular mechanism underlying these physiological and gene 
Development Program of the National Research Foundation (NRF) funded by the Korean government (MSIT) (2019R1A2C2005453).

\section{Authors' contributions}

Conceptualization: Hong SH.

Data curation: Kim JH, Rasaei R.

Formal analysis: Kim JH, Rasaei R.

Methodology: Kim JH, Rasaei R.

Validation: Kim JH, Rasaei R, Park S.

Investigation: Park S, Na S, Kim JY.

Writing - original draft: Rasaei R.

Writing - review \& editing: Hong SH.

\section{Ethics approval}

All animal experiments were approved by the Institutional Animal Care and Use Commitment of Kangwon National University (KW-180809-2). expression changes in diabetic lung tissues, and the therapeutic strategies that may be derived from them, remain to be elucidated.

Administration of mesenchymal stem cells (MSCs) is one promising strategy for the treatment of diabetic complications (Rasaei et al., 2020). Increasing evidence demonstrates that perivascular stem cells (PVCs), known as a precursor of MSCs, exhibit regenerative potential in various disease models, including traumatic brain injury, uterine injury, Achilles tendon rupture, muscle atrophy, and spinal cord injury (Cao et al., 2017; Park et al., 2020). More recently, we demonstrated that PVCs regulate hematopoietic differentiation in a development-specific manner and suppress inflammasome activation during inflammatory responses in murine and human macrophages (Kim et al., 2019; Jeong et al., 2020). These findings suggest that PVCs could be a therapeutic option for treating diabetic complications. In fact, PVCs were found to accelerate wound healing in a diabetic rat and partially rescued hyperglycemia-induced alterations in bone marrow hematopoietic composition (Kim et al., 2018). However, the therapeutic efficacy of PVCs on physiological and gene expression changes in the diabetic lung has not been reported. In the present study, we aimed to determine gene expression changes in the mouse lung due to streptozotocin (STZ)-induced DM. Furthermore, we evaluated the effects of human PVCs on gene expression changes in the diabetic lung.

\section{MATERIALS AND METHODS}

\section{Animals}

C57BL/6J mice were purchased from Dooyeol Biotech (Seoul, Korea) and housed in a specific pathogen-free facility. All animal experiments were approved by the Institutional Animal Care and Use Commitment of Kangwon National University (KW-180809-2). Male C57BL/6J mice (20-22 $\mathrm{g}, 8$ to 10 weeks) were intraperitoneally injected with $50 \mathrm{mg} / \mathrm{kg} \mathrm{STZ} \mathrm{(S0130,} \mathrm{Sigma-Aldrich,} \mathrm{St.}$ Louis, MO, USA) daily for 5 consecutive days to induce type $1 \mathrm{DM}$. After induction of diabetes, mice were intravenously administered with PVC-CM $(40 \mu \mathrm{g} / 100 \mu \mathrm{L})$ daily for 6 weeks.

\section{Immunofluorescence staining}

Immunofluorescence staining was performed as previously described (Kim et al., 2019). For immunofluorescence staining, 4- $\mu \mathrm{m}$ thick lung sections were dewaxed with xylene and rehydrated with a gradient of ethanol. The sections were subjected to antigen retrieval with a citrate buffer bath ( $\mathrm{pH}$ 6) at boiling temperature and then blocked for endogenous peroxidase activity. After rinsing with phosphate buffered saline (PBS), the sections were incubated with primary antibodies against $\alpha$-SMA (Santa Cruz Biotechnology, SC-53015), Zbtb16 (LS Bio, LS-c334349), Adrb2 (Abcam, ab182136), and Amigo2 (LS Bio, LS-c404504) overnight at $4^{\circ} \mathrm{C}$. The sections were washed with PBS and incubated with secondary GFP- or RFP-labelled antibodies for 30 min. Nuclei were counterstained with DAPI (Abcam, ab104139). Immunofluorescence images were captured by fluorescence microscopy (IX-51, Olympus, Tokyo, Japan).

\section{Microarray and data analysis}

Total RNA was extracted from lung tissues using the RNeasy Mini Kit (Qiagen, 74106, Valencia, CA, USA). RNA purity and integrity were evaluated using the Agilent 2100 Bioanalyzer (Agilent Technologies, Santa Clara, CA, USA). Microarray analysis has been performed as previously reported (Kim et al., 2017). 


\section{RNA extraction and real-time quantitative PCR}

Total RNA was extracted from lung tissues using an RNeasy Mini kit (Qiagen, Duesseldorf, Germany) and cDNA was synthesized using the TOPscrip ${ }^{\text {TM }}$ RT DryMIX (Enzynomics, Daejeon, Korea). PCR amplification was performed using a Step One Plus real-time PCR system (Applied Biosystems, Warrington, UK) with TOPreal ${ }^{\mathrm{TM}}$ qPCR 2X PreMIX (Enzynomics). Relative expression was normalized against GAPDH expression by the $\Delta \Delta \mathrm{Ct}$ method. Sequences of primers used in this study are listed in Table 1.

\section{Preparation of PVC-CM}

Human PVCs were obtained from human umbilical cords as previously described. This study was approved by the institutional review board of Kangwon National University Hospital (201406-003-010). All participants provided written informed consent. PVC-CM was prepared as previously described (Jeong et al., 2020). Briefly, PVCs were incubated with serum-free $\alpha$-MEM for $24 \mathrm{hrs}$ and filtered through a $0.22 \mu \mathrm{m}$ filter. Filtered medium was concentrated using an Amicon Ultra-15 Centrifugal Filter Units (Millipore) and stored at $-80^{\circ} \mathrm{C}$ until use.

\section{Statistical analysis}

Values for all measurements are presented as the mean \pm SD. Statistical significance was determined using student's t-test, with $p<0.05$ was considered statistically significant.

\section{RESUTLS}

\section{Gene expression changes in the lungs of diabetic mice}

To investigate the histological and gene expression changes in the lungs following long-term exposure to hyperglycemic conditions, a diabetic mouse model was established in which STZ $(50 \mathrm{mg} / \mathrm{kg})$ was administered for 5 consecutive days. Fig. 1A). Histological analysis showed that hyperglycemia resulted in alveolar wall thickening and a reduction in alveolar air space (Data not shown). We next investigated global gene expression in the lungs of STZ-induced diabetic mice with or without treatment of PVC-conditioned medium (CM). Unsupervised hierarchical clustering showed that the global expression patterns of lung tissue were significantly altered by long-term hyperglycemic condition and partially reversed by PVC-CM treatment (Fig. 1B). A total of 172 genes (72 upregulated and 100 downregulated) were significantly altered in the

Table 1. A list of primers for real time qPCR

\begin{tabular}{|c|c|c|c|c|}
\hline Genes & & Sequence 5' to 3' & Product size (bp) & $\operatorname{Tm}\left({ }^{\circ} \mathrm{C}\right)$ \\
\hline \multirow[t]{2}{*}{ Adrb2 } & $\mathrm{F}$ & GGACAACCTCATCCCTAA & 170 & 60 \\
\hline & $\mathrm{R}$ & AGAGTAGCCGTTCCCATA & & \\
\hline \multirow[t]{2}{*}{ Amigo2 } & $\mathrm{F}$ & CTCGTTGCTGATCTTTTGGT & 80 & 60 \\
\hline & $\mathrm{R}$ & ACCACAGGCGACAGGTATAG & & \\
\hline \multirow[t]{2}{*}{$K I k 1 b 22$} & $\mathrm{~F}$ & CAGCAAAAGCTTCCCTCATC & 108 & 60 \\
\hline & $\mathrm{R}$ & TAAGTCGGCCCCAGTAGGTA & & \\
\hline \multirow[t]{2}{*}{ Zbtb16 } & $\mathrm{F}$ & TGTGAACTCTGCGGAAAACG & 70 & 60 \\
\hline & $\mathrm{R}$ & CCGCTGAATGAGCCAGTAAA & & \\
\hline \multirow[t]{2}{*}{ Gapdh } & $\mathrm{F}$ & GTTGTCTCCTGCGACTTCA & 184 & 60 \\
\hline & $\mathrm{R}$ & GGTGGTCCAGGGTTTCTTA & & \\
\hline
\end{tabular}


A

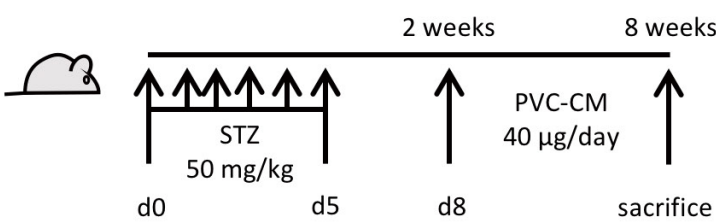

B

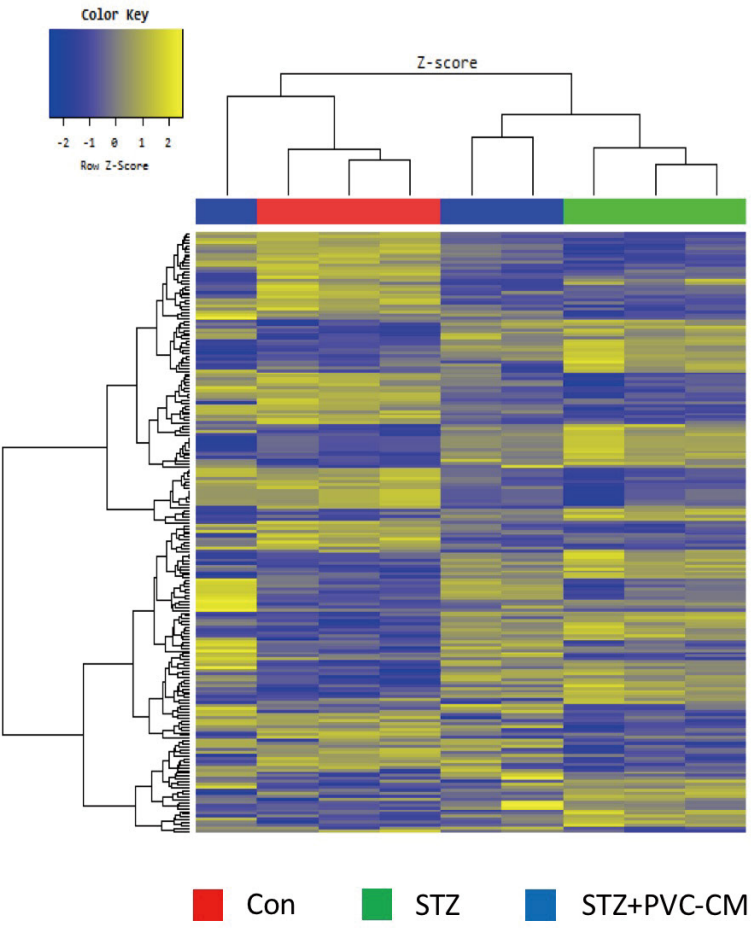

C

GO Biological process (Con vs STZ)

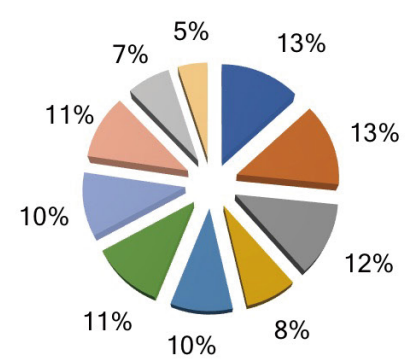

- single-organism process

vellular process

wingle-organism cellular process

- positive regulation of biological process

- response to stimulus

mbiological regulation

vegulation of cellular process

- regulation of biological process

w positive regulation of cellular process

w programmed cell death

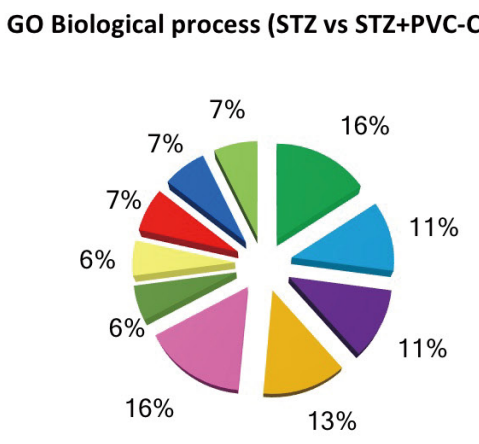

immune system process

- immune response

- regulation of immune system process

positive regulation of response to

stimulus

regulation of response to stimulus

positive regulation of tumor necrosis factor production

positive regulation of tumor necrosis

factor superfamily cytokine production

- immune response-activating signal

transduction

- immune response-regulating signaling

pathway

positive regulation of response to

external stimulus

Fig. 1. Gene expression is altered in the lungs of diabetic mice. (A) A schematic of the PVC-CM treatment protocol after induction of hyperglycemia using STZ. (B) Unsupervised hierarchical clustering analysis of cDNA microarray data from lung tissues of Con, STZ-, and STZ+PVC-CM-treated mice. The color spectrum from blue to yellow indicates low to high expression. (C) Results of gene ontology (GO) analysis of genes differentially regulated between Con and STZ and between STZ and STZ+PVC-CM. Differentially expressed genes (DEGs) were filtered using the selection criteria of fold-change ${ }^{3} \pm 1.5$. PVC, perivascular stem cell; CM, conditioned medium; STZ, streptozotocin; PVC, perivascular stem cell.

STZ group compared with the control. There were also 39 differentially expressed genes (DEGs) (23 upregulated and 16 downregulated) found in the lungs of the STZ group compared with the STZ+PVC-CM group (Table 2). To compare the biological relevance of these DEGs, gene ontology (GO) analyses were performed using total expression data from control, STZ, and STZ+PVC lungs. The main GO categories that included DEGs between the control vs STZ groups were response to cellular process, response to stimulus, and programmed cell death. In addition, a number of general GO terms were identified, including 39 that were associated with differential expression between the STZ and STZ+PVC-CM groups. Of these GO terms, two were characterized by genes related to immune processes and regulation of responses to stimulus (Fig. 1C). These results indicate that long-term hyperglycemia induces gene expression changes in lung tissues that can be partially reversed by PVC treatment.

\section{Expression of Adrb2, Amigo2, and Ztbt16 in the lungs of STZ-induced diabetic mice}

To identify potential target genes associated with hyperglycemia-induced changes in lung 
Table 2. A list of significantly up- and down-regulated genes in STZ versus STZ+PVC-CM

\begin{tabular}{|c|c|c|c|}
\hline Genes & PVC/STZ (fc) & mRNA accession & $p$-value \\
\hline Tcrb-J & 2.206327 & AY665740 & 0.01902 \\
\hline Ighj2 & 2.197561 & ENSMUST00000103429 & 0.00794 \\
\hline Ly96 & 2.005956 & NM_001159711 & 0.01508 \\
\hline Bank1 & 1.994702 & NM_001033350 & 0.02426 \\
\hline Cd19 & 1.914308 & NM_009844 & 0.04525 \\
\hline$C d 79 b$ & 1.849544 & NM_001313939 & 0.03283 \\
\hline$B / k$ & 1.818237 & NM_007549 & 0.00698 \\
\hline $\operatorname{Trgj2}$ & 1.765133 & OTTMUST00000134814 & 0.01139 \\
\hline $\operatorname{lghv3-4}$ & 1.734837 & OTTMUST00000130557 & 0.02912 \\
\hline Ccr7 & 1.721632 & NM_001301713 & 0.02418 \\
\hline Gimap9 & 1.679874 & NM_174960 & 0.01245 \\
\hline Cd180 & 1.659398 & NM_008533 & 0.01516 \\
\hline Ikzf3 & 1.648157 & NM_011771 & 0.04019 \\
\hline Olfr478 & 1.623668 & NM_146734 & 0.02565 \\
\hline Gm14144 & 1.610773 & XR_375105 & 0.01264 \\
\hline Gypa & 1.593726 & NM_010369 & 0.03349 \\
\hline Gsdmc2 & 1.569582 & NM_001168274 & 0.00557 \\
\hline Stk26 & 1.568639 & NM_001313744 & 0.04848 \\
\hline Gm4924 & 1.561515 & $X R \_871890$ & 0.00998 \\
\hline$M s 4 a 4 a$ & 1.552988 & NM_001310331 & 0.01764 \\
\hline$M s 4 a 6 b$ & 1.549311 & NM_027209 & 0.03698 \\
\hline Pf4 & 1.549135 & NM_019932 & 0.00755 \\
\hline Ccr5 & 1.539285 & NM_009917 & 0.0178 \\
\hline Lrrc36 & -1.500673 & NM_001033371 & 0.03294 \\
\hline Dnah7a & -1.508286 & NM_001252070 & 0.03129 \\
\hline Gm17216 & -1.511401 & ENSMUST00000130872 & 0.04972 \\
\hline Gm22887 & -1.523011 & ENSMUST00000178586 & 0.03763 \\
\hline Gm23975 & -1.528846 & ENSMUST00000082685 & 0.01758 \\
\hline Mir1192 & -1.554173 & NR_035423 & 0.02731 \\
\hline Mir1938 & -1.587280 & NR_035459 & 0.0356 \\
\hline Ly6c1 & -1.609003 & NM_001252056 & 0.04903 \\
\hline Cebpd & -1.650718 & NM_007679 & 0.02894 \\
\hline Ft/1 & -1.656437 & NM_010240 & 0.04951 \\
\hline Gm25006 & -1.663671 & ENSMUST00000083813 & 0.0331 \\
\hline$n-R 5 s 29$ & -1.786047 & ENSMUST00000082836 & 0.00459 \\
\hline Gm22023 & -1.789149 & ENSMUST00000104354 & 0.00045 \\
\hline Snord14e & -2.056380 & NR_028275 & 0.02531 \\
\hline Gm20481 & -2.083602 & ENSMUST00000173680 & 0.04559 \\
\hline
\end{tabular}

PVC, perivascular stem cell; STZ, streptozotocin; fc, fold change.

tissues, we first selected 19 up- and downregulated genes from the STZ group where expression could be reversed by PVC-CM treatment (Fig. 2A). Among the group of upregulated genes, qPCR analysis confirmed that the expression pattern of adhesion molecule with $\mathrm{IgG}$-like domain 2 (Amigo2), beta 2-adrenergic receptor (Adrb2), and zinc finger and BTB domain containing 16 (Zbtb16) were identical between the cDNA microarray data (Fig. 2B). Moreover, the Klk1b22 
A

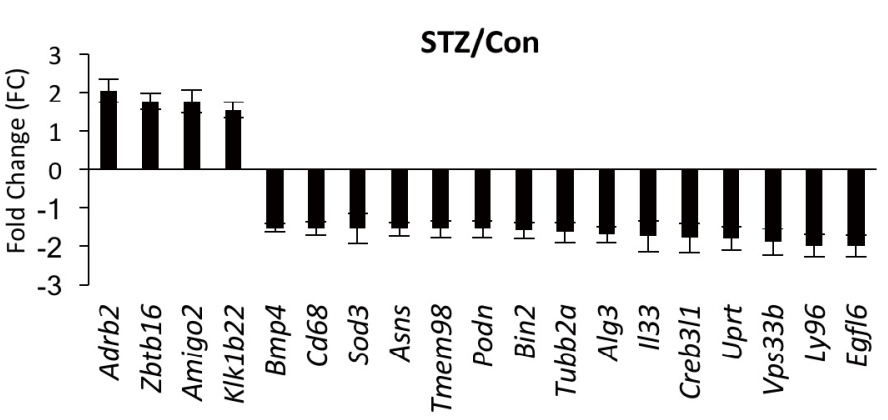

STZ+PVC-CM/STZ

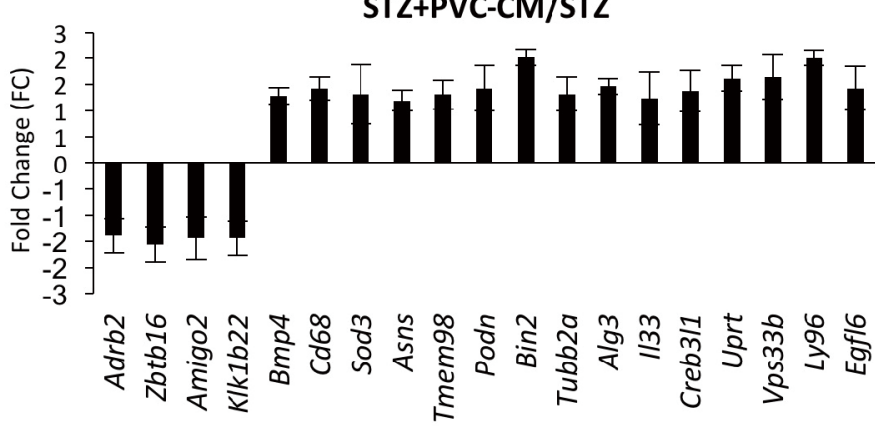

B
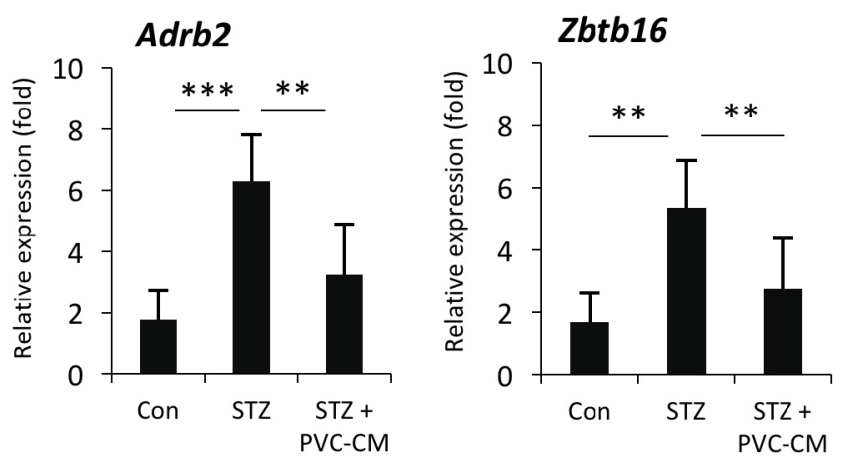

Amigo2

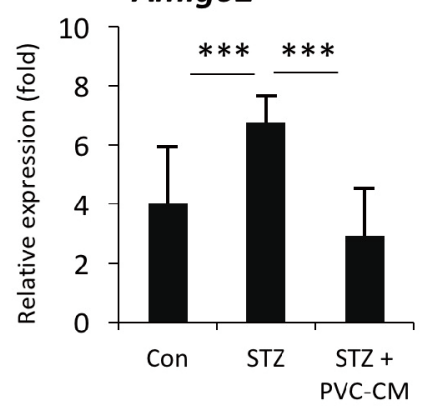

KIk1b22

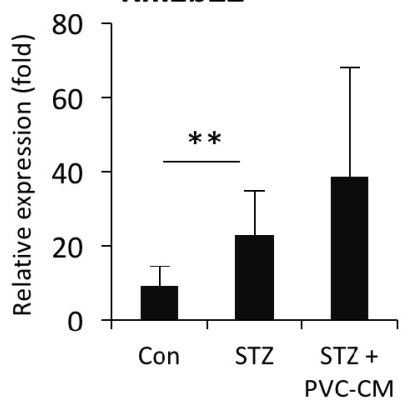

Fig. 2. PVCs reverse upregulated expression of Amigo2, Adrb2, and Zbtb16 in the lung of diabetic mice. (A) The graph shows the hyperglycemiaregulated genes reversed by PVC-CM treatment in diabetic mice lung. (B) qRCR analysis was performed to validate the expression pattern of four genes upregulated in the lung of diabetic mice. Error bars indicate the mean $\pm \mathrm{SD} .{ }^{* *} p<0.01,{ }^{* * *} p<0.001$. PVC, perivascular stem cell; CM, conditioned medium.

gene was upregulated significanty in the STZ group compared to the control but not reversed by PVC-CM treatment (Fig. 2B). Immunostaining of lung tissues demonstrated that Adrb2 and Ztbt16 were localized mainly to the apical membrane of bronchioles, while Amigo2 was detected primarily in endothelial cells of pulmonary vessels (Fig. 3). Consistent with the PCR results, hyperglycemic conditions stimulate enhanced expression of these proteins. These findings suggest that hyperglycemia mediates cell type-specific regulation of Adrb2, Amigo2, and Ztbt16, which could be useful indicators of diabetic injury in lung tissues.

\section{DISCUSSION}

In the present study, we found that long-term hyperglycemia induced alterations in gene expression, as well as histological changes, in the lung. The number and extent of gene expression changes in diabetic lung tissue were moderate compared with findings in other diabetic tissues, including in the kidney, skeletal muscle, and lens, suggesting that the lung is more resistant to hyperglycemic stress than other organ tissues. Importantly, we found that PVCs could partially reverse hyperglycemia-induced gene expression changes in lung gene expression, thereby demonstrating that they can be an alternative for treating diabetic complications in various tissues, including the lung.

This study demonstrated that Adrb2, Amigo2, and Ztbt16 are hyperglycemia-regulated genes in the lung. Although a few studies have reported whether the function and expression of these 

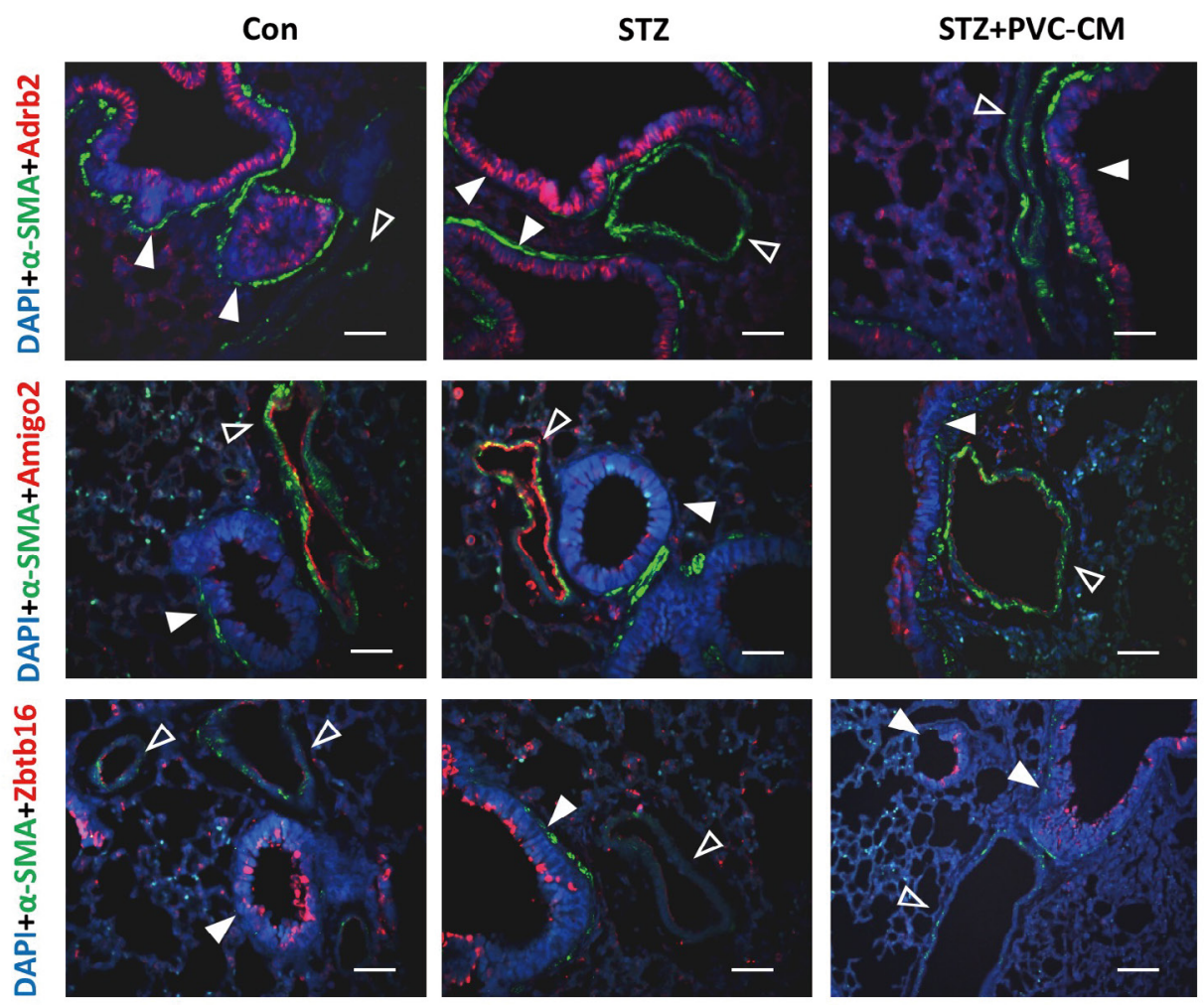

Fig. 3. Immunostaining for Adrb2, Amigo2 and Zbtb16 in the lungs of STZ-induced diabetic mice. Immunofluorescence staining for Adrb2 (red), Amigo2 (red), Zbtb16 (red), and SMA (green) in lung sections from the Con, STZ, and STZ+PVC-CM groups. Nuclei were counterstained with DAPI (blue). Closed arrowheads indicate bronchioles while open arrowheads indicate vessels. Scale bars, $50 \mu \mathrm{m}$. STZ, streptozotocin; PVC, perivascular stem cell; CM, conditioned medium.

genes are associated with histological and physiological changes in diabetic lung tissue, evidence suggests that these genes are potentially useful as prognostic markers to predict lung injury in a diabetic state (Prior et al., 2011; Al-Goblan et al., 2014; Park et al., 2015). Accumulating evidence has demonstrated that Adrb2 is expressed in many cell types in the lung, and it has been considered a critical pharmacologic target in the management of asthma and chronic obstructive pulmonary disease (COPD) due to a correlation between Adrb2 polymorphisms and reduction of lung function (Kim et al., 2009). In addition, significantly higher levels of ADRB2 mRNA were observed in the bronchial mucosa of patients with severe COPD and asthma compared with those displaying mild disease. Given the strong relationship between DM and lung diseases, investigation of downstream signaling activation of $A d r b 2$ and its polymorphisms in diabetic lung tissue may provide a better understanding of the role of this gene in terms of its physiological and gene expression changes in diabetic lung tissue. Long-term hyperglycemia leads to reduced lung function by increasing vascular permeability, suggesting that DM could elevate the risk of tumor metastasis. DM also increased immune cell infiltration in multiple organs and promoted inflammation, mediated by altered expression of several adhesion molecules, including intercellular adhesion molecule 1, vascular cell adhesion molecule 1, and P-selectin, on endothelial cells to augment leukocyte binding (Gu et al., 2013; Pawelczyk et al., 2017; Rasaei et al., 2020). Amigo2, an anti-apoptotic adhesion molecule, is known to be expressed in endothelial cells and involved in angiogenesis and vascular remodeling in pathological situations. Thus, upregulation of Amigo2 in pulmonary vessel endothelial cells of diabetic lung tissue could be involved in promoting influx of immune cells and angiogenesis. These 
findings suggest that targeting Adrb2 and Amigo2 may provide opportunities for the development of novel strategies to prevent or ameliorate hyperglycemia-induced lung injury.

In this study, we identified hyperglycemia-regulated genes in the diabetic mouse lung. However, it remains to be explored whether these genes play a crucial role in developing pulmonary complications and are regulated in the lungs of DM and pulmonary fibrosis patients.

\section{REFERENCES}

Al-Goblan AS, Al-Alfi MA, Khan MZ (2014) Mechanism linking diabetes mellitus and obesity. Diabetes Metab Syndr Obes 7:587-591.

Bissel GJ (2015) Brain changes in T1DM: A microvascular complication? Nat Rev Endocrinol 11:447-448.

Cao Y, Gang X, Sun C, Wang G (2017) Mesenchymal stem cells improve healing of diabetic foot ulcer.J Diabetes Res 2017:9328347.

Ehrlich SF, Quesenberry CP, Jr., Van Den Eeden SK, Shan J, Ferrara A (2010) Patients diagnosed with diabetes are at increased risk for asthma, chronic obstructive pulmonary disease, pulmonary fibrosis, and pneumonia but not lung cancer. Diabetes Care 33:55-60.

Gu HF, Ma J, Gu KT, Brismar K (2013) Association of intercellular adhesion molecule 1 (ICAM1) with diabetes and diabetic nephropathy. Front Endocrinol (Lausanne) 3:179.

Jeong S, An B, Kim JH, Han HW, Kim JH, Heo HR, Ha KS, Han ET, Park WS, Hong SH (2020) BMP4 and perivascular cells promote hematopoietic differentiation of human pluripotent stem cells in a differentiation stage-specific manner. Exp Mol Med 52:56-65.

Kim J, Cha S, Lee MY, Hwang YJ, Yang E, Choi D, Lee SH, Cheon YP (2019) Chronic and low dose exposure to nonlyphenol or di (2-ethylhexyl) phthalate alters cell proliferation and the localization of steroid hormone receptors in uterine endometria in mice. Dev Rep 23:263-275.

Kim JY, Lee JY, Ha KS, Han ET, Park WS, Min CK, Hong SH (2018) Perivascular cells and NADPH oxidase inhibition partially restore hyperglycemia-induced alterations in hematopoietic stem cell and myeloid-derived suppressor cell populations in the bone marrow. Int J Stem Cells 12:63-72.

Kim J, Song H, Heo HR, Kim JW, Kim HR, Hong Y, Yang SR, Han SS, Lee SJ, Kim WJ, Hong SH (2017) Cadmium-induced ER stress and inflammation are mediated through C/EBPDDIT3 signaling in human bronchial epithelial cells. Exp Mol Med 49:e372.

Kim J, Kim WJ, Ha KS, Han ET, Park WS, Yang SR, Hong SH (2019) Perivascular stem cells suppress inflammasome activation during Inflammatory responses in macrophages. Int J Stem Cells 12:419-429.

Kim WJ, Oh YM, Sung J, Lee YK, Seo JB, Kim NK, Kim TH, Huh JW, Lee JH, Kim EK, Lee JH, Lee SM, Lee S, Lim SY, Shin TR, Yoon HI, Kwon SY, Lee SD (2009) CT scanningbased phenotypes vary with $\mathrm{ADRB} 2$ polymorphisms in chronic obstructive pulmonary disease. Respir Med 103:98-103.

Kolahian S, Leiss V, Nurnberg B (2019) Diabetic lung disease: Fact or fiction? Rev Endocr Metab Disord 20:303-319.

Lunteren E, Moyer M, Spiegler S (2014) Alterations in lung gene expression in streptozotocininduced diabetic rats. BMC Endocr Disord 14:5.

Mohamed J, Nazratun Nafizah AH, Zariyantey AH, Budin SB (2016) Mechanisms of diabetesInduced liver damage: The role of oxidative stress and inflammation. Sultan Qaboos Univ Med J 16:e132-e141. 
Park H, Lee S, Shrestha P, Kim J, Park JA, Ko Y, Ban YH, Park DY, Ha SJ, Koh GY, Hong VS, Mochizuki N, Kim YM, Lee W, Kwon YG (2015) AMIGO2, a novel membrane anchor of PDK1, controls cell survival and angiogenesis via Akt activation.J Cell Biol 211:619-637.

Park M, Hong SH, Park SH, Kim YS, Yang SC, Kim HR, Noh S, Na S, Lee HK, Lim H, Lyu SW, Song H (2020) Perivascular stem cell-derived Cyclophilin A improves uterine environment with Asherman's syndrome via HIF1 $\alpha$-dependent angiogenesis. Mol Ther 28:1818-1832.

Pawelczyk M, Kaczorowska B, Baj Z (2017) The impact of hyperglycemia and hyperlipidemia on plasma P-selectin and platelet markers after ischemic stroke. Arch Med Sci 13:1049-1056.

Pitocco D, Fuso L, Conte EG, Zaccardi F, Condoluci C, Scavone G, Incalzi RA, Ghirlanda (2012) The diabetic lung: A new target organ? Rev Diabet Stud 9:23-35.

Prior SJ, Goldberg AP, Ryan AS (2011) ADRB2 haplotype is associated with glucose tolerance and insulin sensitivity in obese postmenopausal women. Obesity (Silver Spring) 19:396-401.

Rasaei R, Kim E, Kim JY, Na S, Kim JH, Heo J, Shin DM, Choi SS, Hong SH (2020) Regulation of JAM2 expression in the lungs of streptozotocin-induced diabetic mice and human pluripotent stem cell-derived alveolar organoids. Biomedicines 8:E346.

Rask-Madsen C, King GL (2013) Vascular complications of diabetes: Mechanisms of injury and protective factors. Cell Metab 17:20-33.

Talakatta G, Sarikhani M, Muhamed J, Dhanya K, Somashekar BS, Mahesh PA, Sundaresan N, Ravindra PV (2018) Diabetes induces fibrotic changes in the lung through the activation of TGF-beta signaling pathways. Sci Rep 8:11920. 
